UB-ECM-PF 92/5

March 1992

\title{
DIFFERENTIAL RENORMALIZATION OF MASSIVE QUANTUM FIELD THEORIES
}

\author{
Peter E. Haagensen 周 and José I. Latorre \\ Departament d'Estructura $i$ Constituents de la Matèria \\ Facultat de Física, Universitat de Barcelona \\ Diagonal 647, 08028 Barcelona, Spain
}

\begin{abstract}
We extend the method of differential renormalization to massive quantum field theories, treating in particular $\lambda \varphi^{4}$-theory and QED. As in the massless case, the method proves to be simple and powerful, and we are able to find, in particular, compact explicit coordinate space expressions for the finite parts of two notably complicated diagrams, namely, the 2-loop 2-point function in $\lambda \varphi^{4}$ and the 1-loop vertex in QED.
\end{abstract}

*e-mail: HAGENSEN@EBUBECM1

${ }^{\dagger}$--mail: LATORRE@EBUBECM1 
Differential renormalization (DR)[1] is a coordinate space renormalization procedure which removes the divergences of bare amplitudes by writing these as derivatives of less singular functions and then prescribing the derivatives to be integrated by parts with all surface terms discarded. The singularities of amplitudes that did not allow for Fourier transformation into momentum space are eliminated and one ends up with finite, renormalized amplitudes that satisfy renormalization group equations. The standard and simplest example of the procedure is for the 1-loop 4-point "bubble" diagram in massless $\lambda \varphi^{4}$, where the following identity is used:

$$
\frac{1}{x^{4}}=-\frac{1}{4} \square \frac{\ln x^{2} M^{2}}{x^{2}} \quad x \neq 0 .
$$

$M$ is an integration constant which will become the subtraction scale of the renormalized amplitude. If we prescribe the laplacian to act only after integration by parts, then the r.h.s. above has a finite Fourier transform, and in fact defines the renormalized value of the (divergent) Fourier transform of the 1.h.s.. Generally, one can say that differential renormalization provides a prescription to continue distributions defined almost everywhere (i.e., bare amplitudes, undefined at a finite number of singular points) into bona fide distributions defined everywhere (renormalized amplitudes).

The procedure exemplified above has been carried out at higher loops and in different models $[1,2,3]$. One can furthermore check explicitly the form of the divergences being subtracted by examining the surface terms which contain them, thus verifying that the method indeed corresponds to a counterterm subtraction procedure [4].

In this Letter, we extend the method to allow for the inclusion of masses in an exact treatment. At the outset, it is clear that the presence of masses should not interfere with the method since the renormalization procedure is related to short-distance singularities whereas masses only change the long-distance behavior of correlators. In fact, one could simply take a pragmatic attitude and expand all massive propagators around zero mass and then proceed to use standard massless differential renormalization. However, the question remains whether massive field theories are amenable to a treatment exact in the mass parameter at each order in perturbation theory. We find that our expectations are entirely fulfilled. In analogy to the massless case, renormalization is accomplished through the use of (massive) differential renormalization identities and an integration by parts prescription. We treat QED at one loop and $\lambda \varphi^{4}$-theory at two loops and, most notably, we are able to give in closed form the full renormalized expressions for 
the 2-loop "setting sun" diagram of $\lambda \varphi^{4}$ and the 1-loop vertex of QED, two results of considerable difficulty of calculation in standard momentum space treatments. In the end, we shall discuss the general features of our modus operandi.

First of all, let us recall the euclidean propagator for a massive scalar particle in four dimensions,

$$
\Delta(x, m)=\frac{1}{4 \pi^{2}} \frac{m K_{1}(m x)}{x}
$$

where $K_{1}$ is a modified Bessel function. Up to permutations of external legs the only 1-loop diagram contributing to the 4-point function in $\lambda \varphi^{4}$ theory is:

$$
\Gamma_{\text {bare }}^{(4)}(x)=\frac{\lambda^{2}}{2}\left(\frac{1}{4 \pi^{2}} \frac{m K_{1}(m x)}{x}\right)^{2}
$$

At short distances, this has the same log divergence as the corresponding massless diagram; in the spirit of differential renormalization we look for an expression that corresponds to the massive generalization of Eq.(1). We find:

$$
\frac{m^{2} K_{1}^{2}(m x)}{x^{2}}=\frac{1}{2}\left(\square-4 m^{2}\right) \frac{m K_{0}(m x) K_{1}(m x)}{x}+\pi^{2} \ln \frac{\bar{M}^{2}}{m^{2}} \delta^{(4)}(x)
$$

where $\bar{M} \equiv 2 M / \gamma$ and $\gamma=1.781072 \ldots$ is the Euler constant. The contact term has been added in order to give a well-defined massless limit to the r.h.s. above, coinciding in fact with Eq.(1). We shall further analyze this later on, and in fact we will see that Eq.(4) can be seen as a prototype in our treatment, since it presents all the basic guiding elements in working out massive DR identities in general. The renormalized 1-loop 4-point function, $\Gamma_{R}^{(4)}(x, M)$, is gotten by simply substituting the above in Eq.(3).

The one-loop beta function can be obtained from the renormalization group equation

$$
\left(M \frac{\partial}{\partial M}+\beta \frac{\partial}{\partial \lambda}+\gamma_{m} m^{2} \frac{\partial}{\partial m^{2}}-4 \gamma\right)\left[-\lambda \delta^{(4)}(x)+\Gamma_{R}^{(4)}(x, M)\right]=0,
$$

where $\beta(\lambda)$ is the $\beta$-function, $\gamma_{m}(\lambda)$ is the anomalous mass dimension, and $\gamma(\lambda)$ the anomalous dimension of $\varphi$. Dropping terms of higher order in $\lambda$ (viz., $\gamma_{m}$ and $\gamma$ ), the result for the $\beta$-function is:

$$
\beta(\lambda)=3\left(\frac{\lambda}{16 \pi}\right)^{2}
$$

with the three permutations of the $s, t$ and $u$ channels having been added. Our result for the bubble diagram can be easily Fourier transformed. The result in momentum space is:

$$
\tilde{\Gamma}_{R}^{(4)}(p)=\frac{\lambda^{2}}{32 \pi^{2}}\left[\ln \frac{\bar{M}^{2}}{m^{2}}-\sqrt{1+\frac{4 m^{2}}{p^{2}}} \ln \frac{\sqrt{1+\frac{4 m^{2}}{p^{2}}}+1}{\sqrt{1+\frac{4 m^{2}}{p^{2}}}-1}\right]
$$


which agrees with the standard result in textbooks [5]. Note the appearance of the 2-particle threshold $4 m^{2}$ in the operator which was extracted in Eq.(4). This will be a recurring feature in what follows. We have also worked out this amplitude for two different masses running along the two lines in the loop; we do not present it here, but rather just mention that the result generalizes the one above and, as expected, the threshold $\left(m_{1}+m_{2}\right)^{2}$ appears instead of $4 m^{2}$.

A far less trivial example showing the power of differential renormalization is given by the computation of the 2-loop correction to the 2-point function (often called the "setting sun"). The complexity of the finite parts of this diagram is such that they are not presented in standard reviews of $\lambda \varphi^{4}$ renormalization. The bare expression for the diagram is:

$$
\Gamma_{\text {bare }}^{(2)}(x)=\frac{\lambda^{2}}{6}\left(\frac{1}{4 \pi^{2}} \frac{m K_{1}(m x)}{x}\right)^{3} .
$$

This bare amplitude is quadratically divergent and thus requires the extraction of two laplacians in order to have a good Fourier transform. Little effort is needed to verify the identity that leads to the following renormalized value of the above diagram:

$$
\begin{aligned}
\Gamma_{R}^{(2)}(x, M)= & \frac{\lambda^{2}}{96\left(4 \pi^{2}\right)^{3}}\left[\left(\square-9 m^{2}\right)\left(\square-m^{2}\right)\left(m^{2} K_{0}(m x) K_{1}^{2}(m x)+m^{2} K_{0}^{3}(m x)\right)\right. \\
& \left.+2 \pi^{2} \ln \frac{\bar{M}^{2}}{m^{2}}\left(\square+a m^{2}\right) \delta^{(4)}(x)\right]
\end{aligned}
$$

This result is by itself a remarkable application of differential renormalization. It corresponds to a closed and compact expression for the renormalized 2-loop 2-point function. Its Fourier transform is complicated and we will not work it out here. Again, we note the appearance of the 3-particle production threshold as a healthy sign of the procedure. Further, the second operator takes the form $\left(\square-m^{2}\right)$, which vanishes on mass shell. The coefficient of the $\square \delta^{4}(x)$ term is fixed as in the previous example by the requirement of a smooth massless limit and this, in turn, fixes $\gamma(\lambda)$ in the renormalization group equation to its standard value

$$
\gamma(\lambda)=\frac{1}{12}\left(\frac{\lambda}{16 \pi}\right)^{2} .
$$

On the other hand, the coefficient $a$ of $m^{2} \delta^{(4)}(x)$ is not fixed by a strict massless limit, which is to be expected, since it corresponds to a mass subtraction, and fixes the (scheme-dependent) $\gamma_{m}$ function in the renormalization group equation. In particular, $a$ can be chosen to be -1 so that the whole 2 -loop contribution vanishes on mass shell. 
Though we do not intend to present an exhaustive computation of the perturbative expansion of massive $\lambda \varphi^{4}$, let us comment on a few more diagrams. There are only two more diagrams contributing at two loops to the 4-point function. The first one, usually called the "ice cream cone", though extremely difficult in momentum space, is easily calculated along the lines we have sketched above. The second one, consisting of two 4-point 1-loop bubbles attached together (and thus, the "double bubble") is not as straightforward due to the presence of a convolution.

We now turn to the computation of 1-loop diagrams in QED f. The (massive) fermion and photon propagators are, respectively:

$$
S(x, m)=\frac{1}{4 \pi^{2}}(\not \partial-m) \frac{m K_{1}(m x)}{x},
$$

and

$$
\Delta_{\mu \nu}(x)=\frac{1}{4 \pi^{2}} \frac{\delta_{\mu \nu}}{x^{2}}
$$

this latter one being given in Feynman gauge. The $\bar{\psi} A \psi$ vertex has the value $i e \gamma_{\mu}$. The 1-loop fermion self-energy then reads:

$$
\Sigma_{\text {bare }}(x)=\left(\frac{i e}{4 \pi}\right)^{2} \frac{1}{x^{2}} \gamma_{\mu}(\not \partial-m) \frac{m K_{1}(m x)}{x} \gamma_{\mu} .
$$

The mass piece above has a logarithmic short-distance divergence, while the derivative term is linearly divergent at short distances. These divergences are eliminated by the following massive DR identity:

$$
\frac{m K_{1}(m x)}{x^{3}}=\frac{1}{2}\left(\square-m^{2}\right) \frac{K_{0}(m x)}{x^{2}}+\pi^{2} \ln \frac{\bar{M}^{2}}{m^{2}} \delta^{(4)}(x),
$$

where again the contact term is determined such as to give a well-defined massless limit to the r.h.s. (and again equal to the corresponding massless identity). The final, renormalized expression for the fermion self-energy will then be:

$$
\begin{aligned}
\Sigma_{R}(x, M)= & \frac{e^{2}}{32 \pi^{2}}\left[\left(\square-m^{2}\right)\left((\not \partial+4 m) \frac{K_{0}(m x)}{x^{2}}+\frac{m^{2}}{2} \frac{\not x K_{0}(m x)}{x^{2}}\right)\right. \\
& \left.+2 \pi^{2} \ln \frac{\bar{M}^{2}}{m^{2}}(\not \partial+b m) \delta^{(4)}(x)\right] .
\end{aligned}
$$

The non-local piece of this amplitude vanishes identically on mass-shell due to the operator $\left(\square-m^{2}\right)$, as expected. In analogy to the previous case, the $\not \partial \delta^{4}(x)$ contact

\footnotetext{
${ }^{\ddagger}$ For a complete set of momentum space techniques to compute one-loop diagrams in gauge theories, see [6].
} 
term is fixed by the massless limit (and leads to the standard value for the anomalous dimension of the fermionic field), whereas the $m \delta^{4}(x)$ term is not. We leave the coefficient $b$ undetermined, and again it is clear that a particular choice, viz. $b=-1$, makes the whole amplitude vanish on mass-shell. The Fourier-transformed, momentum space amplitude can be obtained fairly straightforwardly, and we do not present it here.

The bare 1-loop vacuum polarization is:

$$
\Pi_{\mu \nu}^{\text {bare }}(x)=-\left(\frac{i e}{4 \pi^{2}}\right)^{2} \operatorname{tr}\left(\gamma_{\mu}(\not \partial-m) \frac{m K_{1}(m x)}{x} \gamma_{\nu}(-\not \partial-m) \frac{m K_{1}(m x)}{x}\right) .
$$

By standard manipulations with Bessel functions and the use of a massive DR identity, Eq. (4), we find the following renormalized vacuum polarization at one loop:

$$
\begin{aligned}
\Pi_{R \mu \nu}(x, M)= & -\frac{e^{2}}{24 \pi^{4}}\left(\partial_{\mu} \partial_{\nu}-\delta_{\mu \nu} \square\right)\left[( \square - 4 m ^ { 2 } ) \left(\frac{m K_{0}(m x) K_{1}(m x)}{x}\right.\right. \\
& \left.\left.+\frac{m^{2}}{2}\left(K_{1}^{2}(m x)-K_{0}^{2}(m x)\right)\right)+2 \pi^{2} \ln \frac{\bar{M}^{2}}{m^{2}} \delta^{(4)}(x)\right] .
\end{aligned}
$$

As expected, the result is automatically transverse and shows the presence of the 2particle production threshold in the operator coming in front of the non-local piece of the amplitude.

We finally turn to the 1-loop vertex. Its bare value is:

$$
V_{\mu}^{\text {bare }}(x, y)=\left(\frac{i e}{4 \pi^{2}}\right)^{3} \gamma_{\rho}(\not \partial-m) \frac{m K_{1}(m x)}{x} \gamma_{\mu}(-\not \partial-m) \frac{m K_{1}(m y)}{y} \gamma_{\rho} \frac{1}{(x-y)^{2}} \text {. }
$$

In the above, the only divergent piece is the one containing the two derivatives:

$$
V_{\mu}^{d i v}(x, y)=-\left(\frac{i e}{4 \pi^{2}}\right)^{3} \gamma_{\rho} \not \partial \frac{m K_{1}(m x)}{x} \gamma_{\mu} \not \partial \frac{m K_{1}(m y)}{y} \gamma_{\rho} \frac{1}{(x-y)^{2}} .
$$

We renormalize this by an identical procedure used in the massless case [1], i.e., by integrating the two derivatives by parts onto $1 /(x-y)^{2}$, and separating that into a (finite) traceless piece and a (divergent) trace piece. The divergence, thus isolated in the trace, is then renormalized with massive DR identity Eq.(4). The final result reads:

$$
\begin{aligned}
& V_{\mu}^{R}(x, y, M)=\left(\frac{i e}{4 \pi^{2}}\right)^{3}\left[2 \gamma _ { b } \gamma _ { \mu } \gamma _ { a } \left(\frac{\partial}{\partial x^{a}}\left[\frac{m K_{1}(m x)}{x} \frac{\partial}{\partial y^{b}}\left(\frac{m K_{1}(m y)}{y}\right) \frac{1}{(x-y)^{2}}\right]\right.\right. \\
& \left.-\frac{\partial}{\partial y^{b}}\left[\frac{m^{2} K_{1}(m x) K_{1}(m y)}{x y} \frac{\partial}{\partial x^{a}} \frac{1}{(x-y)^{2}}\right]-\frac{m^{2} K_{1}(m x) K_{1}(m y)}{x y}\left(\partial_{a} \partial_{b}-\frac{1}{4} \delta_{a b} \square\right) \frac{1}{(x-y)^{2}}\right) \\
& -2 \pi^{2} \gamma_{\mu}\left(\square-4 m^{2}\right) \frac{m K_{0}(m x) K_{1}(m x)}{x} \delta^{(4)}(x-y)-4 \pi^{4} \gamma_{\mu} \ln \frac{\bar{M}^{2}}{m^{2}} \delta^{(4)}(x) \delta^{(4)}(x-y) \\
& \left.+4 m \frac{1}{(x-y)^{2}}\left(\frac{\partial}{\partial y^{\mu}}-\frac{\partial}{\partial x^{\mu}}-\frac{m}{2} \gamma_{\mu}\right)\left(\frac{m^{2} K_{1}(m x) K_{1}(m y)}{x y}\right)\right] .
\end{aligned}
$$


This concludes our results. We have extended the method of differential renormalization to massive $\lambda \varphi^{4}$ and QED, and the results we present here, though not intended to be exhaustive, should indicate both the feasibility of, and the guidelines for, the treatment of massive fields at higher loops and in different models. These guidelines basically are: a) the presence of an equal number of K-functions on both sides of massive DR identities; b) a direct connection to massless differential renormalization, through the substitution of $\ln x^{2} M^{2}$ in this latter case for $K_{0}(m x)$; c) the appearance of the appropriate particle production thresholds in the differential operators present in massive DR identities, and d) the appearance of contact terms, due to the requirement of existence of the massless limit for renormalized distributions. These features have been of central importance in helping us find all the renormalized amplitudes presented here.

Two final comments are in order regarding contact terms in massive DR identities. Firstly, the presence of these terms may seem puzzling, since they vanish at points where massive DR identities are valid mathematical equations, i.e., for $x \neq 0$. However, insofar as these identities are prescriptions for extending (i.e., renormalizing) distributions, they should be understood as identities everywhere, including at contact, and specific contact terms will then determine precisely and uniquely the value of the extended (i.e., renormalized) distributions at those points. Finally, it is also worth noting that contact terms encode the entire renormalization group freedom present in the renormalization procedure. Our choice has been to fix contact terms in order to agree with the massless case, which corresponds to a wave function renormalization prescription, and to leave undetermined the mass subtraction, though an on-mass-shell scheme has been indicated.

Acknowledgments - We would like to thank D.Z. Freedman for ongoing discussions. This work was supported in part by CAICYT grant AEN90-0033 and by the EEC Science Twinning Grant SCI-000337. P.H. also acknowledges a grant from the Ministerio de Educación y Ciencia, Spain.

\section{References}

[1] Freedman, D.Z., K. Johnson and J.I. Latorre, Nucl.Phys. B, in press.

[2] Haagensen, P.E., Mod.Phys.Lett. A7(1992)893. 
[3] Freedman, D.Z., G. Grignani, K. Johnson and N. Rius, "Conformal Symmetry and Differential Regularization of the 3-Gluon Vertex", MIT preprint CTP\#1991.

[4] Freedman, D.Z., R. Muñoz-Tapia and X. Vilasís-Cardona, manuscript in preparation.

[5] Ramond, P., Field Theory, A Modern Primer, 2nd ed., Addison-Wesley(1990).

[6] 't Hooft, G. and M. Veltman, Nucl.Phys. B153(1979)365. 